\title{
Cellular Automaton for Kidney Branching Morphogenesis
}

\author{
AFSHIN POORKHANALIKOUDEHI, KARL-HEINZ ZIMMERMANN \\ Institute of Embedded Systems \\ Hamburg University of Technology \\ 21071 Hamburg \\ GERMANY
}

\begin{abstract}
Epithelium is a complex component in the mammalian kidney that has a highly branched duct system. Branching morphogenesis has a hierarchy structure in the ureteric bud and produces the collecting duct tree through repetitive processes. Epithelial and mesenchymal cells surround the tips of growing branches, and their cellular reactions adjust the ureteric bud branching. Mesenchymal cells produce a small protein called glial cellline derived neurotrophic factor (GDNF) that connects to te Rearranged in Transfection (RET) receptors on the surface of epithelial cells. The identified reactions are a necessity for the normal branching growth and their roles exist for using biological features in the proposed model.

This paper presents an agent-based model based on cellular automaton for kidney branching in ex-vivo using the features that are expressed as artificial patterns in algorithms. This model extending the groundbreaking approach of Lambert et al. is flexible in features and high compatibility with experimental data. Mesenchymal cells and RET receptors are also expressed as mathematical patterns in the algorithms. The growth mechanism is determined by the growth factor, which indicates the epithelial cell branch when its cell division depends on the local concentration growth factor. Cell division occurs when the level of stimulus growth factor exceeds the threshold. Comparison shows that the model mimics experimental data with high consistency and reveals the dependence between growth factor parameters and features. Results indicate the superiority of compatibility with nature when compared with the model mentioned above.
\end{abstract}

Key-Words: - Cellular automaton, Agent-based model, Organogenesis, Mathematical modeling of branching morphogenesis, Morphology.

Received: April 27, 2021. Revised: October 3, 2021. Accepted: October 25, 2021. Published: November 13, 2021.

\section{Introduction}

Kidney is a complex bean-shaped organ with a highly branched tree-like structural system. This tree structure is organized during embryogenesis and is known as branch morphogenesis. The process of branch formation is essential for the growth of the kidney as an organic system with a similar architectural structure such as lung, breast, and salivary gland. Normal growth and branching of the urinary bud (UB) are also necessary for the growth of complete nephrons, and their low number is a risk factor for developed hypertension and chronic kidney disease in humans [6, 23, 36]. To understand how such disorders occur, the basic questions about the growing kidney are addressed including how to start the branching, how to adjust it, and how to stop and control the branching [25]. Answering these questions is biologically time-consuming and expensive due to the complex structure of the kidney. Biologically derived experiments examine a limited aspect of morphogenesis, which is further complicated by a combination of hypotheses. Simulations can be performed to support and measure the true final effects of alter- native conditions, multiple hypotheses, and to generate new predictions that can be tested during the experiment $[8,11,46]$.

Kidney branching begins biologically with the outgrowth of the primary urinary bud (UB) from the nephric duct to the adjacent metanephric mesenchyme (MM), and the UB bifurcates during embryonic growth [36, 43]. Glial cell-line derived neurotrophic factor (GDNF) is a secretory protein that plays a key role in UB branch morphogenesis. It transmits information via the RET receptor tyrosine kinase and the Gfr $\alpha 1$ co-receptor [13, 14, 36]. GDNF is primarily expressed in metanephric mesenchymal (MM) cells by E11.5 (embryonic day 11.5) [25] and then by nephron-producing cells (mesenchymal cap) that completely enclose the UB tip of kidney growth [8, 21, 36]. RET is described by cells entirely the terminal UB tip, but it is not considered by cells in the trunks [31, 36]. In fact, the tubular sections behind the tips are long, narrow, and distinct to organize the aggregation ducts. GDNF is also known as a chemoattractant and stimulates the growth of epithelial cells [26]. The experiments have accomplished that the cell-cell contact between mesenchyme and 
epithelium is not required for branching [2, 35]; it is sufficient when epithelial cells (ECs) are exposed to dissolved factors caused by MM. The mechanical forces between EC and mathematical modeling show that this mechanism can have an impressive effect and produce some results in branching morphogenesis [25, 42].

Many mathematical models have been developed in the field of branching morphogenesis with different aspects such as physical cellular processes [22, 37], molecular processes [40, 46], as well as a combination of both [12]. Recent research has provided general differential equations to describe how EC population dynamics grow at the tips of branching. These tips are controlled by mesenchymal cells [46].

A mathematical model summarizes the discovered dynamics using the ratio of ECs to mesenchymal cells at the branch tips again. Von Neumann (1966) first developed the idea of a dynamic replicator, a simple agent-based model such as cellular automata (CA). Generalities such as the cellular Potts model (CPM) and various hybrid models are currently used to study and understand different types of biological systems such as biochemical reaction networks, stem cell proliferation, stem cell differentiation, tumor angiogenesis, and metastasis [18, 27, 38].

Our agent-based epithelial cells are organized in a regular, two-dimensional lattice. Their rate of migration and proliferation are regulated by the local distribution of GDNF. Our CA is based on agent epithelial cells realized in a regular, two-dimensional lattice. The rate of their migration and proliferation is regulated by the local distribution of GDNF. Such frameworks are usually simpler and faster to simulate than off-grid models and have fewer limitations on cell movement [5, 33, 34].

RET signaling, mesenchyme, anisotropic, chemotaxis, and GDNF features [25, 36] are considered for our mathematical model as branch-of-class (BOC) meaning different roles for kidney branching are known as classes. The focus is on the "function" and "action" roles for features in the BOC model (Fig. 1). These features will be modeled mathematically and our proposed simulation model is also comparable to experimental data. The local levels of the general growth factor, known as GDNF, act as a proxy for their influence. The CA model approach does not require hypotheses about natural cell-cell forces that have not yet been experimentally proven [25]. Simulation are based on a two-dimensional CA model and uses experimental data from [36]. The main contributions are as follows:

- A new algorithm (CA model) inspired by the Potts model and biological features is proposed to simulate kidney branching morphogenesis.
Moreover, input parameters obtained from the features to perform the simulation are presented.

- The principle of dimensionless GDNF concentration in kidney morphogenesis is combined with the Gaussian probability density function of the features to provide a new energy function known as the probability of the cell division.

- The superiority of the CA model compared to the simulation model of [25] is demonstrated based on the usage of cellular automata.

The remaining sections are organized as follows. In Sect. 2, our cellular automaton model is described. Sect. 3 shows the simulation results and provides a comparison with an existing model. Finally, our model and its prospects are discussed in Sect. 4 .

\section{Cellular Automaton Modeling}

This section briefly describes our CA step by step. Note that in the introduction, the biological features of the BOC model (Fig. 11) were shortly described. It will be explained how to present the parameters as model inputs in order to simulate renal branching by experimental data as data and feature set. In the CA simulation, it will be shown how individual cells depend on GDNF as a steady-state function through the model. This model uses the cellular Potts model (CPM) to encourage neighboring nodes to carry the same label [41]. Biological features are RET signaling, mesenchyme, anisotropic, and chemotaxis [25, 36]. The model is an extended form of a CA model [25] to which the features RET signaling and mesenchyme have been added, and offers a suitable imitation of the biological model. It works on a rectangular Euclidean lattice in which each cell is represented as a subset of lattice sites. CPM is a generalization of the Ising model for multiple discrete states that is useful for some phenomena such as cell migration, clustering, and cell branch growth [9, 41] as well as volume and surface constraints [3] $]$.

\subsection{BOC Model}

The illustration in Fig. 11 shows two paths "function" and "action" in order to reach CA. In the function path, a differential equation is solved and the results known as dimensionless GDNF concentration are used in the algorithms. In the action path, the computational process is given by algorithms for use in the simulation (Sect. 2.3). The CA algorithms use probabilities $P_{m}$ and $P_{c d}$ to determine cell movement and cell division, respectively. Dimensionless GDNF concentration $g$ is involved which will be detailled in Sect. 2.3. In the model, the input data sets are biological images and require image processing techniques which are described next. 


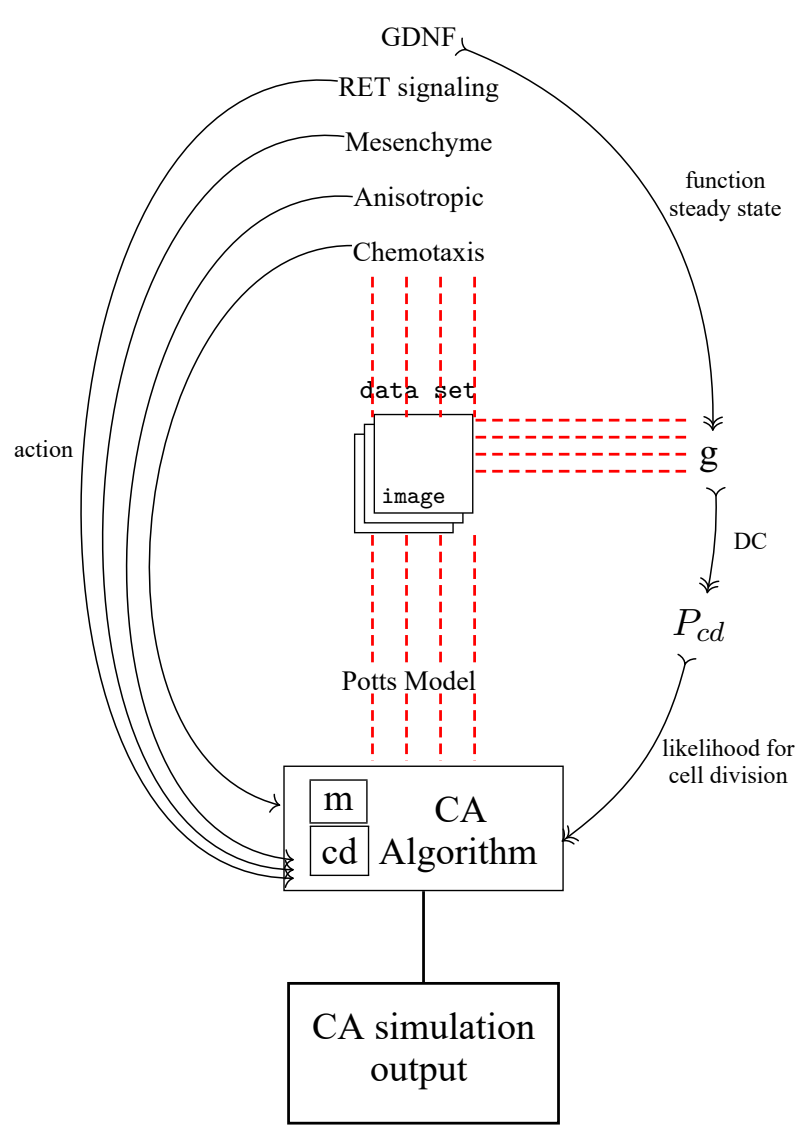

Fig. 1: The branch of class (BOC) model has a simulation part and CA output. The simulation features are GDNF, RET signaling, mesenchyme, anisotropic, and chemotaxis. The data set is given by biological images of kidney branching growth, also $m$ and $c d$ are considered as cell movement and cell division, respectively. DC refers to dimensionless concentration.

\subsection{Data Set and Image Processing}

Dynamic branching occurs in ex-vivo to evaluate the role of Etv4 in UB tip cell behavior, and the role of the RET/Etv4 pathway is provided in UB cell behavior during branch morphogenesis [36]. E11.5 (Embryonic day 11.5) kidneys were cultured and timelapse imaged by fluorescence microscopy. Kidney dissected and stained with a specific antibody from a transgenic embryo at E11.5 verifying the expression of green fluorescent protein (GFP) signal throughout the UB from Hoxb7/CreGFP. Kidneys were cultured and two-dimensional (2D) images in which the identity of each cell in a labeled clone has marked a related video (S1 Movie in [36]) from 0 to $59 \mathrm{~h}$. S1 movies are intended as experimental data, and twodimensional anatomical images can also be used.

Video has been processed to extract some characteristics such as total cells, number of epithelial cells (ECs), terminal cells of the branch, and number of

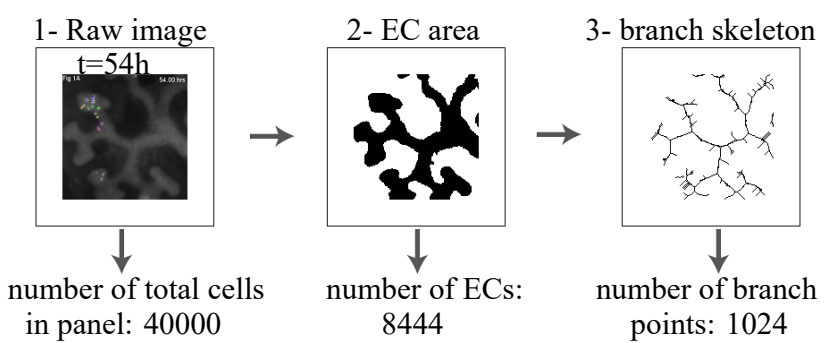

Fig. 2: The image processing workflow includes a raw image of the video in step 1, the extracted epithelial cell (EC) in step 2, and the branch skeleton extracted in step 3.

branch cells. It provides experimental data that two samples are sufficient to establish the model. Image processing and data analyses have been done by using Matlab. Raw images were extracted at a specific time from the video that are taken as experimental data. EC region and branch skeleton extracted from the raw images are used for the distribution surface and the terminal cells of the branch (Fig. 2). Branch skeletons and terminal cells are performed by the "bwmorph" function in Matlab [4]. This function also provides suitable binary and morphological operators such as "endpoints", "majority", "skeleton" and "thin".

EC area is monitored as a measure of the change in the number of compatible cells for comparison with experimental data. Comparisons with similar simulation models as well as the similarity of our CA model with its biological prototype are made over time. The position of all ECs is considered in the overtime simulation, so a differential equation based on the time needs to be solved as described in the following section.

\subsection{CA Model and Algorithms}

A two-dimensional CA is considered in which the individual behavior of ECs depends on the location of neighbors, the local concentration of GDNF, and association with the nodes in the lattice (Fig. 3). Individual behavior of the ECs includes speed, direction, distribution, and consumption of the GDNF. Interactions between cells are based on repeated rules, and behavior can be observed in this way. In the process, equations are used for cellular stimulation that lead to the reproduction of the main features of morphogenesis. The ECs capture a square surface as a domain into $N \times N$ with equal-spaced lattice cells. Either the $\mathrm{EC}$ or the extracellular matrix (ECM) occupies each square lattice. The ECs mass is considered in the center of the domain, and the ECM occupies all other sites at $\mathrm{t}=0$.

GDNF diffusion coefficient has a $2 \mathrm{D}$ reactiondiffusion equation for morphogens [24, 44]]. The quasi-steady state approximation in the two- 


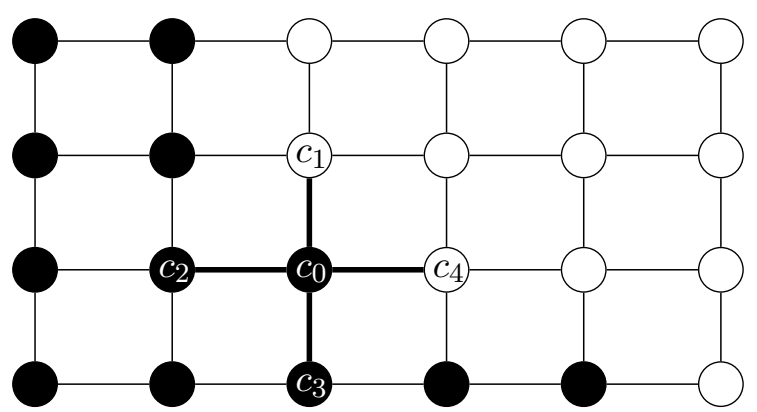

Fig. 3: 2D lattice model with von Neumann neighborhood is shown as UGM. Black cells are ECs and the remaining cells are empty. Above $c_{2}$ and $c_{3}$ are interior cells, $c_{0}$ is a border cell, and $c_{1}$ and $c_{4}$ empty marginal cells.
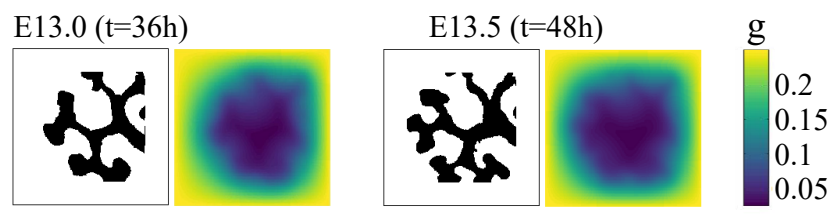

Fig. 4: GDNF level $g$ for images with dimension $200 \times 200$. The letter " $\mathrm{g}$ " is dimensionless GDNF concentration.

dimensional reaction-diffusion equation Eq. (11) [25] is considered. For this, the distribution of the GDNF is $G(x, y, t)$, where $(x, y)$ is the grid point and $t$ denotes time:

$$
\frac{\partial G}{\partial t} \approx 0=D_{G} \nabla^{2} G-\Phi_{G},
$$

where $D_{G}$ is the assumed-constant diffusion coefficient for GDNF, the local rate of GDNF consumption $\Phi_{G}$ is $K_{G} G(x, y, t)$ (with rate parameter $K_{G}$ ) for $t \neq 0$ and is 0 for ECM (at $t=0$ ), and $G$ denotes $G(x, y, t)$.

Eq. (11) is solved using the partial differential equation (PDE) for spatial parameters $x_{l}=\frac{x}{L}, y_{l}=\frac{y}{L}$ with image length $L$, where dimensionless GDNF concentration $0 \leq g \leq 1$, dimensionless diffusion coefficient $d_{g}$, and dimensionless GDNF uptake term $\phi_{g}$ are provided [25]. This equation is solved for our image data set at the given time step (Fig. (4) and uses dimensionless GDNF concentration $g$ in the algorithms (Algs. 1-6). The CA model and finite difference plan are performed on the same separate lattice, so the local GDNF level is considered for updating the rules and its value is at the same lattice point in which the cell is located. Location and rate of GDNF uptake affect the evolution of the GDNF field and this field also influences the movement and behavior of the ECs; dimensionless diffusion coefficient is considered at constant

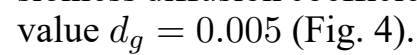

In this model, there are two "actions" $m$ and $c d$ known as cell movement and cell division, respectively (Fig. 1). Cellular individuals follow this simple rule that cells either move $(m)$ or perform cell division $(c d)$ (Alg. 1). It is assumed that cell death is negligible due to the results of preceded experiments [20, 25]. The algorithm starts by solving the reaction-diffusion equation as shown above for the current $\mathrm{EC}$ locations. A random permutation list is used for cell indicators and updates. In the update method, if a cell has empty neighborhood sites (Fig. 3), it is decided to suggest an event $m$ or $c d$ to one of the empty locations. The local concentration of GDNF leads to determine the proposed "action" and is performed in the empty site.

The proposed action is then determined to perform the $m$ or $c d$ event into one of the empty marginal cells. Border cells are considered here as epithelial (black) cells located in the center of the von Neumann neighborhood that have at least one empty neighbor known as marginal cells (Fig. 3). Mesenchyme and RET signal algorithms (Algs. 3 and 4) using neighboring the EC (black) sites around the border cells lead to decisions on the Anisotropic (Alg. 5) or Chemotaxis (Alg. 6). The local GDNF concentration in the kernel algorithm (Alg. 2) determine whether the proposed action, if any, takes place in an empty cell.

Alg. 1 determines whether four adjacent sites in the von Neumann neighborhood (up, down, left, and right) are empty. If so, the "action" for empty cells is performed, which occurs for a move with probability $P_{m}$ or for a cell division with probability $1-P_{m}$. Probability $P_{m}$ is independent of GDNF and is taken to suggest the "action" that may not be performed. Movement $(m)$ is always done, but the cell division $(c d)$ depends on the local level of the GDNF concentration (kernel-simulation algorithm). In Alg. 2, the quantity $P_{c d}$ is the probability of cell division into coordinate $\left(x_{l}, y_{l}\right)$ at time $t$, which comes from the cellular calculations and the Gaussian distribution function (Algs. 3 and 4). In Eq. (2), the quantity $P_{c d}$ known as the energy function is actually the probability of cell division in the location $\left(x_{l}, y_{l}\right)$ at time $t$. For each location at time $t$, there are the local GDNF level $g\left(x_{l}, y_{l}, t\right)$, the border cell $x$ (ECs area), and the local GDNF level $g_{x}\left(x_{l}, y_{l}, t\right)$ in the border cell, denoted by $g, x$, and $g_{x}$, respectively (Fig. 5).

In Eq. (2), the algorithms 3 and 4 need to be performed in advance. Mesenchyme action in Alg. 3 is responsible for the computation of empty cells outside the epithelial cells (ECs). RET signaling action in Alg. 4 is used for computing the Gaussian in $N(0,1)$, and $\varphi($.$) is the PDF with mean \mu=0$ and standard deviation $\sigma=1$ (write $\varphi(. \mid \mu=0, \sigma=1)$ ). In these algorithms, the branching skeleton is taken to extract the variables such as the skeleton endpoint (Sect. 2.2) known as a terminal cell $(t c)$. The vari- 

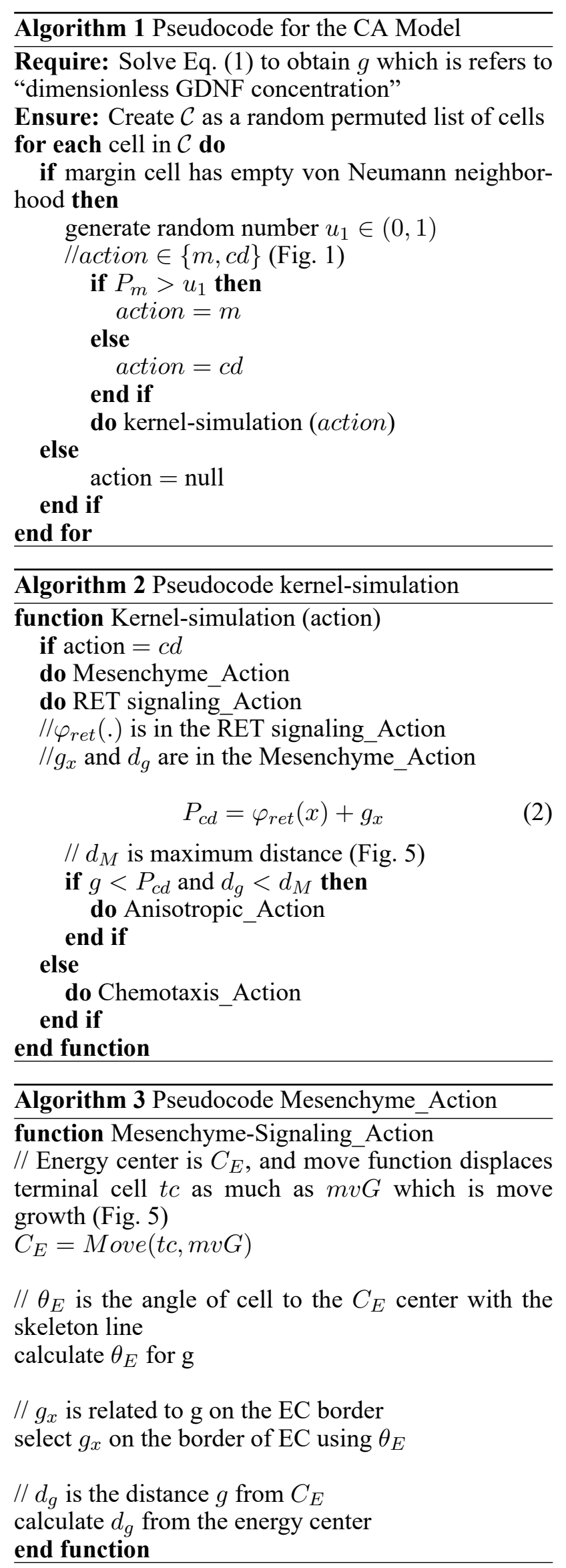
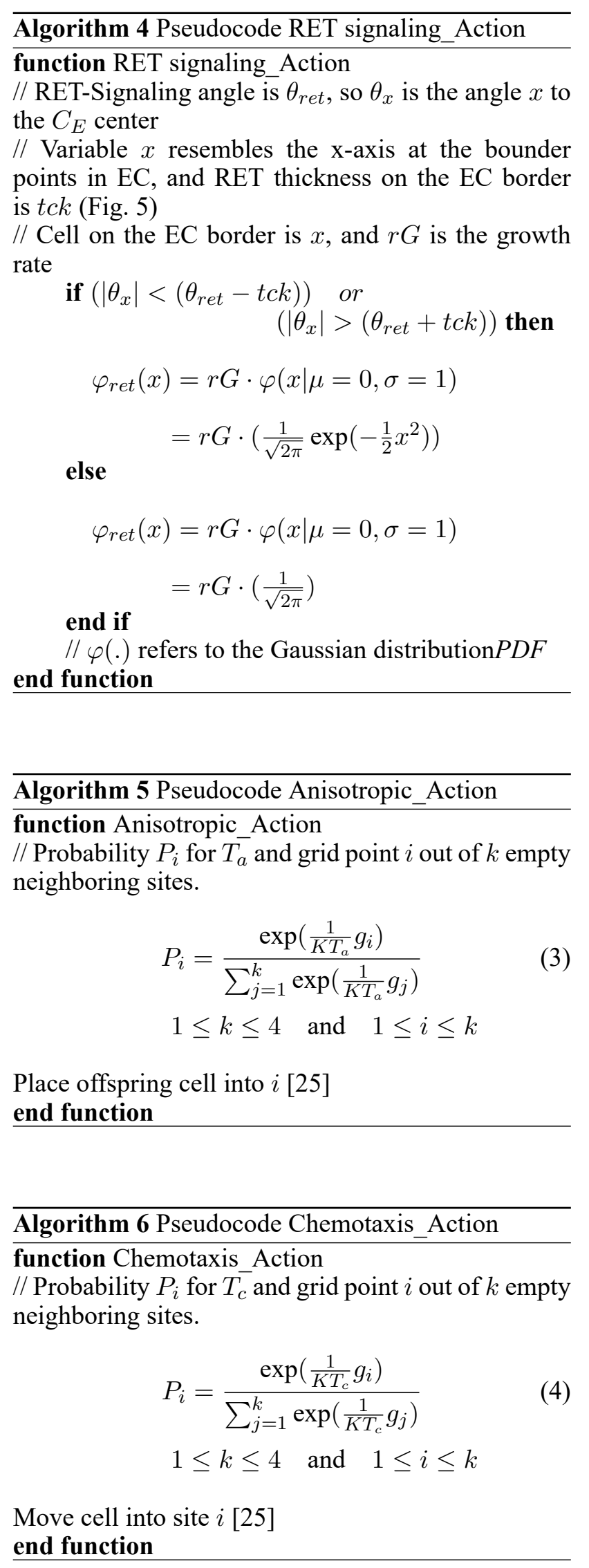

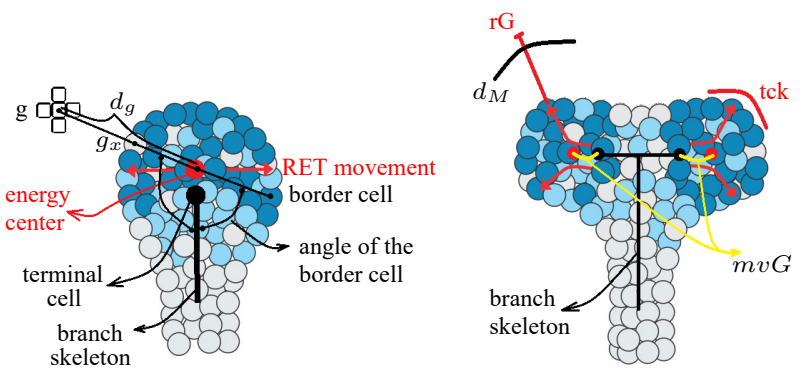

Fig. 5: Energy center $\left(C_{E}\right)$, terminal cell $(t c)$, angle cell $\left(\theta_{E}\right.$ for each cell and $\theta_{\text {ret }}$ for RET movement vector), border cell ( $x$ and $g_{x}$ ), and distance $g$ from $C_{E}$ $\left(d_{g}\right)$, maximum distance from $C_{E}\left(d_{M}\right)$, move growth $(m v G)$, growth rate $(r G)$, and RET thickness (tck) are shown.

ables used in the procedures are illustrated in Fig. 5. Terminal center $t c$ plays a key role and involves the variables growth rate $r G$, angles $\theta_{E}$ and $\theta_{\text {ret }}$, and energy center $C_{E}$. Growth rate $r G>0$ controls the rate of branching development, while angle $\theta_{\text {ret }}$ and the thickness $t c k$ determine the sensitivity to local GDNF level $g$ (Alg. (4). In the absence of quantity $r G$ $(r G=0)$, epithelial cells do not divide.

When the "action" for each cell is set to $m$ or $c d$, it is decided which of the empty neighbors at the grid point will be proliferated by the final action. If there is more than one empty grid point, the GDNF level leads to select bias on the neighboring sites. Mesenchyme, RET signaling, and anisotropic actions (Algs. 3-5) refer to the process of cell division and chemotaxis action (Alg. 6) is related to the movement process. In Eqs. (3-4), the probability $P_{i}$ known as Gibbs measure selects an empty neighboring cell $i$ given by the related equation, where $K$ is a universal constant. In the equations, the quantities $T_{a}$ and $T_{c}$ are the temperature parameters for the chemotaxis and anisotropic algorithms that control the selection sensitivity for the local GDNF concentration $g_{i}$. These algorithms are carried out by using MATLAB (Octave).

\section{Results}

Simulations will be performed for our CA model and a comparison with an existing model will be made.

\subsection{Implementation}

CA simulation model generates branch patterns similar to the ex-vivo kidney explant from the image data set. Raw images are selected from the data set [36] as sample inputs using image processing to extract the EC area (Sect. 2.2). Standard panel size is considered 200 by 200 for processing and the dimensionless GDNF concentration $g$ is calculated for the image data base. CA simulation model requires the param- eter set $\theta=\left(\theta_{r e t}, t c k, r G, d_{M}, P_{m}, m v G\right)$ for implementation. These parameters are RET signaling angle $\theta_{\text {ret }}$, RET thickness on the EC border $t c k$, growth rate $r G$, maximum distance $d_{M}$, probability of cell movement $P_{m}$, and growth move $m v G$. The process is illustrated in Fig. 6. Our model is used to survey the contribution of cell proliferation based on the parameter set for kidney branching. Cell proliferation does not occur in the absence of growth rate $r G$, maximum distance $d_{M}$, and probability $P_{m}$ parameters.

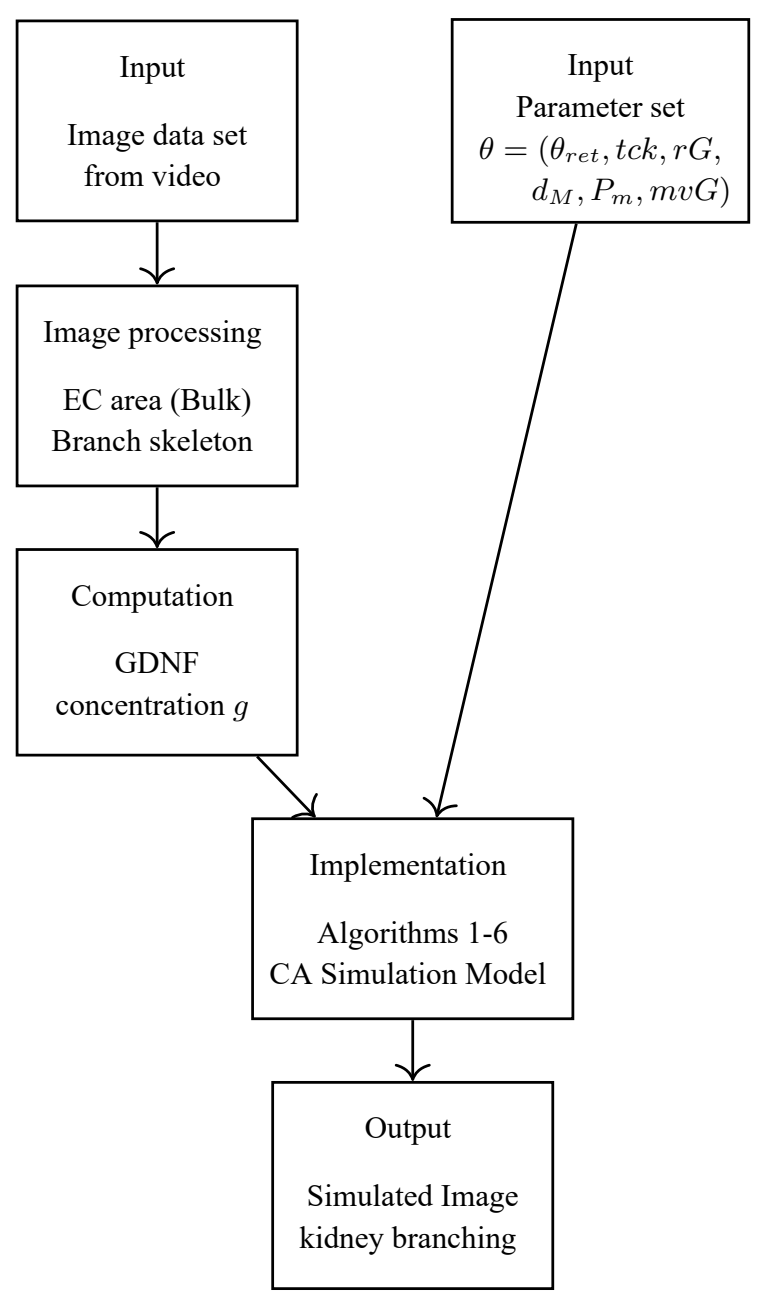

Fig. 6: Processing using input algorithms. One iteration with schematic inputs based on image processing and CA simulation model.

In view of hyperparameter tuning, Iterated Local Search (ILS) [1, 30] is employed, which is a randomized local search method suitable for tuning the parameters used in the algorithms. For this, all parameters are tested for performance within a certain range by changing one parameter value at a time. CA simulation model is iterated 1000 times for any growth time (Fig. 7) and the top 5\% with the smallest distance from normalized area is accepted to compose 


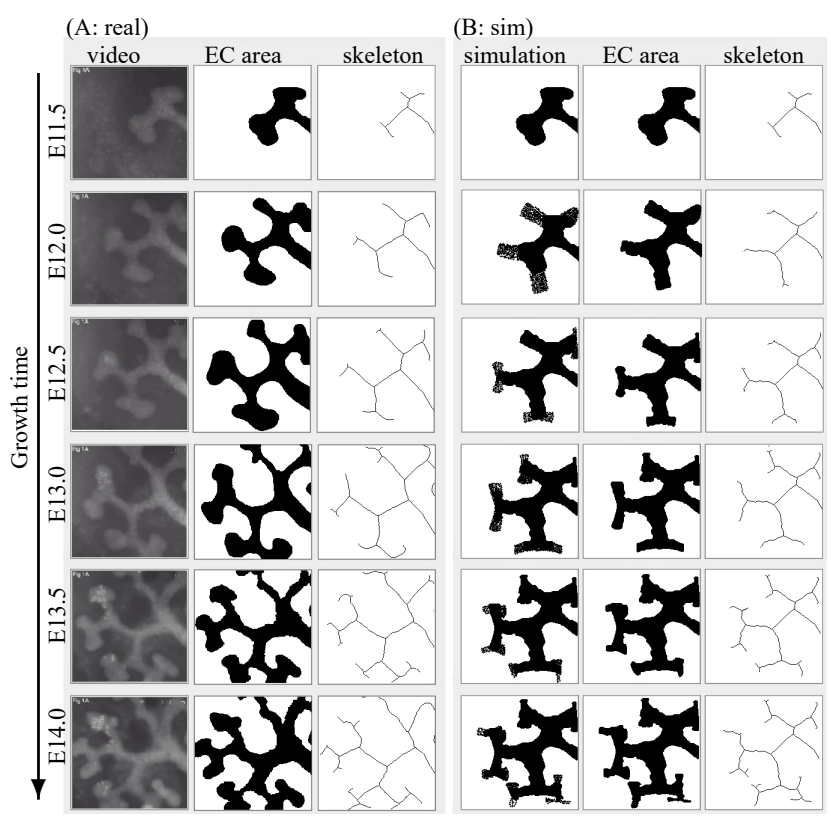

Fig. 7: Comparison of branching patterns related to (A) ex-vivo data set as real and (B) common simulations of our CA model as sim. Image processing techniques (Sect. 2.2) are applied to extract the major shape from the EC areas (center panel) and then to produce the center axis skeletons (right panel). The parameter sets are shown in Tab. 1.

approximate posterior samples. The normalized area is obtained by dividing the number of common cells in both the simulation and biological images by the total number of simulation cells. Hu moment distance (Sect. 3.2) relates simulation and biological model and so provides another criterion for determining the parameters. This measure leads to a similar result in Tab. 1. There is the marginal distribution in the ILS for the parameter set at each growth time, so the high density (mode) parameters of each time are illustrated in the table. In this figure, the posterior distribution of the parameter set summarizes the uncertainty values in the BayesianTools library function of the $\mathrm{R}$ language and is a report for the growth time of E12.0. ILS is performed for other growth times to obtain the parameter set, whose results are summarized in Tab. 1. Distribution of the parameters were chosen because the simulation results show that the range of the parameters is likely to reach the EC area and the branch skeleton to the real explants. All parameters except the parameter set are kept fixed in the table. The CA simulation model is performed on a corresponding parameter set that is more consistent with the experimental data. Compatibility for skeletons and EC areas is presented in Sect. 3.2 as well as a comparison with another model in [25].

CA simulation results are shown in Figs. 7 and 8

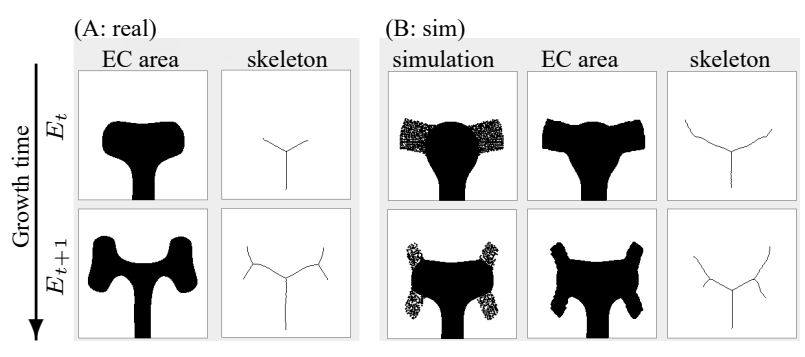

Fig. 8: Comparison of branching patterns related to (A) sample data as real and (B) common simulations of our CA model as sim. Image processing techniques (Sect. 2.2) are applied to produce the axis skeleton. Parameter sets are shown in Tab. 1.

for both ex-vivo data and data samples, respectively. The simulation model reveals that the branching patterns are similar to the specimens identified (Sect. 3.2) in the ex-vivo kidney explant data set [36]. In these figures, the results of common simulation results are compared with the experimental data collected at six growth times. The simulation has significant branching features: the branches are located at both ends of the buds and the secondary branches are followed at the tips of the branches. Simulation explants also create branch events in which two branches emerge from a single tip, events that occur in a growing kidney. CA model takes approximately 10 seconds to generate a single-branch simulation on a desktop computer (Intel (R) Core (TM) i5-7200U CPU, $2.70 \mathrm{GHz}$, 8GB RAM).

\subsection{Model Comparison}

Comparison of CA simulation model called model I with the experimental image data (Sect. 2.2) is conducted by calculating the compatibility of the results in Fig. 7 using a matching function. This procedure is also performed for the agent-based model [25] called model II with the same data set at the corresponding times in Fig. 9. In these figures, the experimental data in (A) and the models' results in (B) are shown at the growth times E11.5 to E14.0. In each model, two columns of EC area (real and simulated) are compared for each growth time as well as two skeleton columns (real and simulated). Compatibility and distance between the two images are computed using $\mathrm{Hu}$ moments.

Hu moment invariants are a set of seven numbers calculated by central moments that are invariant under image transformations. The first six moments are proven to be invariant under translation, scale, rotation, and reflection, while the sign of the seventh moment changes under reflection of the image [45, 47]. Computation of the $\mathrm{Hu}$ moments of the images is shown in Figs. 7 and 9 making use of the HuMoments() function in OpenCV. Since the data are white 
Summary of parameter values

\begin{tabular}{lcccccccc}
\hline Par & Des & $E_{t}$ & $E_{t+1}$ & E12.0 & E12.5 & E13.0 & E13.5 & E14.0 \\
\hline $\mathrm{L}$ & 1 & 200 & 200 & 200 & 200 & 200 & 200 & 200 \\
$d_{g}$ & 2 & 0.005 & 0.005 & 0.005 & 0.005 & 0.005 & 0.005 & 0.005 \\
$g_{\infty}$ & 3 & 1 & 1 & 1 & 1 & 1 & 1 & 1 \\
\hline$\theta_{r e t}$ & 4 & 91.44 & 132.1 & 123.11 & 84.18 & 93.42 & 95.04 & 97.44 \\
$t c k$ & 5 & 8.35 & 1.28 & 2.29 & 6.49 & 4.95 & 5.29 & 2.67 \\
$r G$ & 6 & 0.72 & 0.58 & 0.55 & 0.69 & 0.79 & 0.71 & 0.91 \\
$d_{M}$ & 7 & 68.31 & 66.03 & 59.41 & 22.62 & 29.60 & 17.06 & 21.09 \\
$P_{m}$ & 8 & 0.87 & 0.58 & 0.67 & 0.83 & 0.68 & 0.79 & 0.89 \\
$m v G$ & 9 & 1.62 & 8.81 & 1.97 & 2.80 & 1.59 & 4.10 & 0.81 \\
$T_{a}$ & 10 & 2 & 2 & 2 & 2 & 2 & 2 & 2 \\
$T_{c}$ & 11 & 2 & 2 & 2 & 2 & 2 & 2 & 2 \\
$K$ & 12 & 1 & 1 & 1 & 1 & 1 & 1 & 1 \\
\hline
\end{tabular}

Table 1: Summary of parameter values used in the simulations. Parameter values correspond to Figs. 7 and 8 with different growth times. Parameters are expressed in the Par column. Description of each parameter in the Des column are (1) domain width and height (cell diameters), (2) dimensionless diffusion coefficient, (3) dimensionless diffusion GDNF at boundaries, (4) RET signalling angle, (5) RET thickness on the EC boder, (6) growth rate, (7) maximum distance for growth, (8) probability of selecting move vs division, (9) growth movement of the energy center, (10) anisotropic temperature, (11) chemotaxis temperature, and (12) universal constant.

shapes on a black background, the grayscale images are thresholded to binary. In Tabs. 2 and 3, the log transform $H_{i}=-\operatorname{sign}\left(h_{i}\right) \log \left|h_{i}\right|$ is utilized to establish Hu moments $h_{i}, i=1, \ldots, 7$, for all images in the figures separately in the growth times.

Distance between two images (real and simulated) is computed using $\mathrm{Hu}$ moments. When the distance between the real and the simulated images is small, the images are close in appearance; otherwise, the images are farther away [47]. Function "matchShapes" provided by OpenCV captures two images (contours) and calculates their distance using $\mathrm{Hu}$ moments [16, 45, 47]. There are four types of distance methods: CONTOURS-MATCH-I1, CONTOURS-MATCH-I2, CONTOURS-MATCHI3, and Euclidean distance using Eqs. (5-8), known as distances 1 to 4 , respectively. Let $A$ and $B$ be two images as input for the distance $d(A, B)$, and $H_{i}^{A}$ and $H_{i}^{B}$ be the $i^{\text {th }} \log$-transformed Hu moments for the corresponding images, respectively. Distance methods 1 to 4 are defined as follows:

$$
\begin{aligned}
& d_{1}(A, B)=\sum_{i=1}^{7}\left|\frac{1}{H_{i}^{A}}-\frac{1}{H_{i}^{B}}\right|, \\
& d_{2}(A, B)=\sum_{i=1}^{7}\left|H_{i}^{A}-H_{i}^{B}\right|,
\end{aligned}
$$

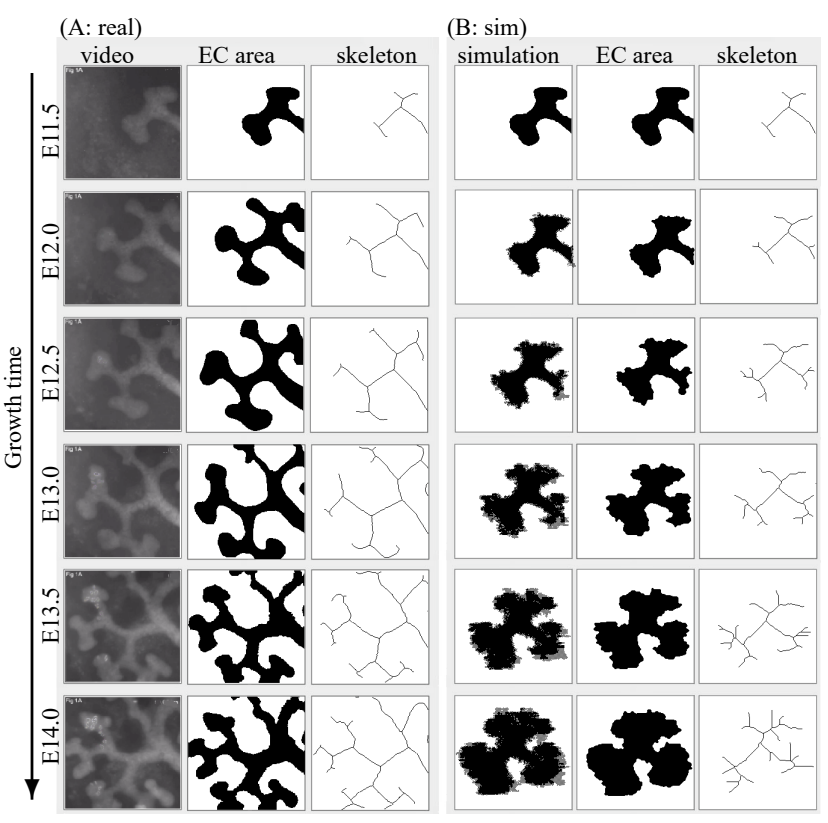

Fig. 9: Comparison of branching patterns related to (A) ex-vivo data set (real) and (B) simulation by CA model (sim) [25]. Image processing techniques (Sect. 2.2) are applied to extract the major shape from the EC areas (center panel) and then to produce the center axis skeletons (right panel). The values of the parameters for generating the simulations are taken from Table S1 in [25].

$$
\begin{gathered}
d_{3}(A, B)=\sum_{i=1}^{7} \frac{\left|H_{i}^{A}-H_{i}^{B}\right|}{\left|H_{i}^{A}\right|}, \\
d_{4}(A, B)=\sqrt{\sum_{i=1}^{7}\left(H_{i}^{A}-H_{i}^{B}\right)^{2}} .
\end{gathered}
$$

Comparison using these four $\mathrm{Hu}$ moments for both image types (real and simulated) in each growth phase (Figs. 7) and 9) is exhibited in Tabs. 4 and 5. The common features of these two tables are summarized in the following. First, the growth times are the same in both tables. Second, there are epithelium area and skeleton in both. Third, different methods of Hu moment distance $d_{1}$ to $d_{4}$ are calculated. Finally, the input images of the biological model are the same for both simulations, which are presented in the tables as the RI (real image) column. In fact, the conditions for calculating the distances are the same in the two simulation models. Tabs. 4 and 5 also represent the distance for model I (our simulation) and model II [25], respectively. However, Hu moment distance in Tab. 4 is much smaller than in Tab. 5, and this is also exhibited in Fig. 10 for different methods in epithelium and skeleton. When the distance 
$\mathrm{Hu}$ moments for the EC areas in models I and II

\begin{tabular}{|c|c|c|c|c|c|c|c|c|}
\hline GT & EI & $H_{1}$ & $H_{2}$ & $H_{3}$ & $H_{4}$ & $H_{5}$ & $H_{6}$ & $H_{7}$ \\
\hline 0 & RI & 2.8963 & 6.9294 & 10.2336 & 11.7921 & -22.866 & 15.2569 & 23.1108 \\
\hline I & s1 & 2.9398 & 6.7984 & 9.9641 & 11.2995 & 22.1770 & 14.7748 & -22.016 \\
\hline [1] & s2 & 2.9945 & 6.6933 & 10.0742 & 11.3656 & -22.326 & -14.884 & -22.172 \\
\hline \multirow{3}{*}{$\stackrel{\sim}{\stackrel{n}{1}}$} & RI & 2.887 & 7.5091 & 9.7105 & 11.271 & -21.781 & -15.228 & -22.290 \\
\hline & s1 & 2.9205 & 6.8602 & 9.6221 & 11.365 & 22.3747 & 14.9676 & -21.879 \\
\hline & s2 & 3.0045 & 6.9363 & 10.0410 & 11.3545 & -22.456 & -14.890 & -22.089 \\
\hline \multirow{3}{*}{$\begin{array}{l}\stackrel{0}{2} \\
\stackrel{\vec{I}}{ }\end{array}$} & RI & 2.8258 & 7.8580 & 9.6606 & 10.3524 & 20.6435 & 14.293 & 20.4272 \\
\hline & s1 & 2.9271 & 7.1105 & 9.6233 & 11.232 & 21.6653 & 15.7541 & -22.453 \\
\hline & s2 & 3.0277 & 7.1832 & 10.0370 & 11.4971 & -22.569 & -15.103 & -22.325 \\
\hline \multirow{3}{*}{$\stackrel{n}{n}$} & RI & 2.8643 & 8.5220 & 9.7031 & 10.5788 & -20. & -14 & 20.8122 \\
\hline & s1 & 2.9338 & 7.1997 & 9.7071 & 11.5025 & 22.2252 & -15.340 & 22.2966 \\
\hline & s2 & 3.0622 & 7.3921 & 10.2032 & 11.7532 & -22.857 & -15.465 & -22.909 \\
\hline \multirow{3}{*}{$\begin{array}{l}\stackrel{\circ}{ \pm} \\
\stackrel{+}{I}\end{array}$} & RI & 2.8998 & 7.9151 & 9.7552 & 11.0457 & -21.565 & -15 & 21.6334 \\
\hline & s1 & 2.9389 & 7.2914 & 9.7330 & 11.8040 & -22.911 & -15.456 & 22.6238 \\
\hline & s2 & 3.1033 & 7.5505 & 10.3146 & 12.0246 & -23.207 & -15.841 & -23.806 \\
\hline
\end{tabular}

Table 2: Calculated values of the $\log$ transform Hu moments $H_{1}$ to $H_{7}$ for the images related to the EC areas. At each growth time (E12.0, E12.5, E13.0, $\mathrm{E} 13.5$, and E14.0), there are three rows, which are real image (RI), simulation 1 (s1), and simulation 2 (s2). The real EC area corresponds to the first panel (A) of Figs. 7 or 9. Simulations 1 and 2 are the simulations in models 1 and 2 of the EC area images from the second panel (B) of Figs. 7 and 9, respectively. At growth time E11.5, due to the uniformity of the images in these figures, the repetitive $\mathrm{Hu}$ moments are eliminated (Tabs. 4-5). GT and EI are growth time and $\mathrm{EC}$ area images, respectively.

is small, the images (real and simulated) are close in appearance [16, 47]. Therefore, our CA simulation model known as model $\mathrm{I}$ is visually closer to the ex-vivo model than the existing model II. This superiority for the EC and skeleton is illustrated in Fig. 10, which indicates that the model I imitates kidney branching with higher precision than the other one; blue area of the figure in model I is much smaller than red area in model II.

First three distances $d_{1}$ to $d_{3}$ behave similarly because they have the same amplitude of change, while the Euclidean distance $d_{4}$ has different behavior because the amplitude of the changes is quite different from the first three. The reason is the range of the domain of the distances (Fig. 10). Hu moments and distances were calculated using the OpenCV module in Python.

\section{Discussion}

An extention of mathematical model of Lambert et al. [25] for the simulation of branching morphogenesis in growing kidney has been presented. This model makes use of knowledge of different branching systems [28, 29, 36, 41] and is the first model to propose renal morphogenesis based on the BOC model. The model also provides a suitable imitation of the bio-
Hu moments for the skeletons in models I and II

\begin{tabular}{|c|c|c|c|c|c|c|c|c|}
\hline GT & SI & $H_{1}$ & $H_{2}$ & $H_{3}$ & $H_{4}$ & $H_{5}$ & $H_{6}$ & $H_{7}$ \\
\hline \multirow{3}{*}{ 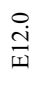 } & RI & 1.5496 & 4.3775 & 5.9394 & 6.8443 & -13.293 & -9.452 & -13.552 \\
\hline & s1 & 1.5603 & 4.2974 & 6.4778 & 6.6681 & 14.0738 & 8.8265 & -13.245 \\
\hline & s2 & 1.6231 & 4.1797 & 5.3151 & 6.5288 & -12.657 & -10.088 & 12.5566 \\
\hline \multirow{3}{*}{ 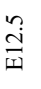 } & RI & 1.4995 & 4.1124 & 6.1077 & 8.1805 & -15.413 & 10.2760 & -15.562 \\
\hline & s1 & 1.5416 & 4.0644 & 5.3111 & 7.3184 & 13.8652 & 9.8121 & -13.724 \\
\hline & $\mathrm{s} 2$ & 1.6409 & 4.1378 & 6.0548 & 7.2123 & -13.897 & -9.319 & -14.182 \\
\hline \multirow{3}{*}{ 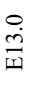 } & RI & 1.4765 & 4.2041 & 5.5228 & 6.9069 & 13.1288 & 9.1046 & 13.8701 \\
\hline & s1 & 1.5613 & 4.2775 & 5.5758 & 7.3414 & 13.9441 & -9.8548 & 3.9571 \\
\hline & $\mathrm{s} 2$ & 1.6288 & 4.27092 & 5.5523 & 7.2869 & -14.558 & -10.216 & 13.7109 \\
\hline \multirow{3}{*}{$\frac{n}{m}$} & RI & 1.5310 & 4.8110 & 5.7900 & 6.8678 & 13.6545 & 9.3362 & 3.2248 \\
\hline & s1 & 1.5879 & 4.5458 & 5.6477 & 7.0888 & -13.543 & -9.7042 & -13.698 \\
\hline & $\mathrm{s} 2$ & 1.6674 & 4.0245 & 6.0351 & 7.9377 & 14.9778 & 10.0320 & 15.2540 \\
\hline \multirow{3}{*}{$\underset{\text { 品 }}{\stackrel{0}{ \pm}}$} & RI & 1.5483 & 4.7315 & 5.6331 & 6.6425 & 12.9246 & 9.0232 & 12.9373 \\
\hline & s1 & 1.6078 & 4.7114 & 5.6672 & 6.9133 & 13.2561 & 9.5487 & -13.537 \\
\hline & s2 & 1.7137 & 4.4499 & 5.5329 & 6.5525 & 12.7976 & 8.8852 & 12.7039 \\
\hline
\end{tabular}

Table 3: Calculated values of the $\log$ transform Hu moments $H_{1}$ to $H_{7}$ for the images related to the skeletons. At each growth time (E12.0, E12.5, E13.0, E13.5, and E14.0), there are three rows, which are real image (RI), simulation 1 (s1), and simulation 2 (s2). The real skeleton corresponds to the first panel (A) of Figs. 7 or 9 . Simulations 1 and 2 are the simulations in models 1 and 2 of skeleton from the second panel (B) of Figs. 7 and 9, respectively. At growth time E11.5, due to the uniformity of the images in these figures, the repetitive Hu moments are eliminated (Tabs. 4-5). GT and SI are growth time and skeleton images, respectively.

logical model. An important advantage of the presented approach is that unlike other methods [28, 46] the process works at cell level. Cell resolution is especially important in determining the concentration threshold of growth factors at which branching occurs. The presented CA simulation model framework allows the description of cells based on tissue morphogenesis. New biophysical mechanisms and signaling pathways can be added to the proposed model which have the possibility to extend to different tissues by distinct types of branching and growth.

There are differences between the dynamics of branching that occurs ex vivo and in vivo [39]. Thus care should be taken when extracting the data image from the former to the latter state. Here, the focus lies on an ex-vivo model due to the availability of newly released image data [36]. CA simulation model is also presented in $2 \mathrm{D}$ and receives its features from this system, which is computationally more tractable than $3 \mathrm{D}$ simulation. In fact, there will be significant barriers to $3 \mathrm{D}$ computing as its topology requires more details than 2D. Thus different mechanisms with more features must be taken into account to update the cell states. The presented simulation model uses the diffusion of growth factors [10] which is sufficient for $2 \mathrm{D}$ data sets, while the growth factors need to be up- 
Matching distance methods in model I

\begin{tabular}{|c|c|c|c|c|c|c|c|}
\hline & Time & RI & SI & $d_{1}$ & $d_{2}$ & $d_{3}$ & $d_{4}$ \\
\hline & E11.5 & & & 0.0 & 0.0 & 0.0 & 0.0 \\
\hline$\frac{\vec{\sigma}}{3}$ & E12.0 & & & 0.0051 & 0.0435 & 0.0150 & 63.764 \\
\hline $\bar{n}$ & E12.5 & & & 0.0039 & 0.0334 & 0.0115 & 53.499 \\
\hline$\overline{\mathscr{J}}$ & E13.0 & & & 0.0122 & 0.1010 & 0.0357 & 42.932 \\
\hline లేّ & E13.5 & & & 0.0082 & 0.0694 & 0.0242 & 43.233 \\
\hline II & E14.0 & & & 0.0045 & 0.0391 & 0.0134 & 1.9566 \\
\hline & E11.5 & & & 0.0 & 0.0 & 0.0 & 0.0 \\
\hline$\frac{\pi}{7}$ & E12.0 & & & 0.0086 & 0.0906 & 0.0182 & 32.917 \\
\hline$\frac{n}{0}$ & E12.5 & & & 0.0210 & 0.0900 & 0.0280 & 29.363 \\
\hline 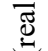 & E13.0 & & & 0.0408 & 0.1582 & 0.0574 & 18.982 \\
\hline 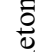 & E13.5 & & & 0.0355 & 0.3222 & 0.0551 & 42.746 \\
\hline है & E14.0 & & & 0.0247 & 0.0795 & 0.0384 & 26.483 \\
\hline
\end{tabular}

Table 4: Comparison is based on four $\mathrm{Hu}$ moments distance methods $d_{1}$ to $d_{4}$. The real and the corresponding simulated images (at each growth time) are two inputs for the match function. Calculation for the epithelium EC areas and skeletons is performed in our CA simulation model (Fig. 7), which is specified in the vertical column description. RI and SI are real images and simulated images, respectively.

dated for the 3D simulation model. The 2D model allows us to study complex mechanisms in a simpler geometric environment before entering 3D dynamics. These details has led to the development of a suitable simulation model that significantly mimics its biological model and is superior to the model of Lambert et al. [25]. Models in dynamic 3D space for tumors [19], fusion of deep learning features [17] and optimization in the simulation model, new distance methods [15] to monitor biological growth, methods such as AABC and ABCDP [7, 32] are based on approximate Bayesian computation for estimating parameters, and more complex hybrid parameters and branching models for other tissues known in organogenesis require further investigation in the future.

\section{References:}

[1] Abualhaija, S., Tahmasebi, N., Forin, D., and Zimmermann, K.H., Parameter Transfer
Matching distance methods in model II

\begin{tabular}{|c|c|c|c|c|c|c|c|}
\hline & Time & RI & SI & $d_{1}$ & $d_{2}$ & $d_{3}$ & $d_{4}$ \\
\hline & E11.5 & & & 0.0 & 0.0 & 0.0 & 0.0 \\
\hline 壱 & E12.0 & & & 0.0113 & 0.0982 & 0.0339 & 54.402 \\
\hline क & E12.5 & & & 0.0135 & 0.1174 & 0.0406 & 1.0330 \\
\hline$\overparen{\Xi}$ & E13.0 & & & 0.0235 & 0.2018 & 0.0714 & 67.537 \\
\hline $\mathbb{E}$ & E13.5 & & & 0.0225 & 0.1978 & 0.0691 & 43.801 \\
\hline II & E14.0 & & & 0.0226 & 0.2035 & 0.0702 & 45.490 \\
\hline & E11.5 & & & 0.0 & 0.0 & 0.0 & 0.0 \\
\hline$\frac{\tilde{\Xi}}{\Xi}$ & E12.0 & & & 0.0400 & 0.2712 & 0.0474 & 26.135 \\
\hline$\vec{n}$ & E12.5 & & & 0.0589 & 0.1668 & 0.0942 & 19.726 \\
\hline 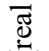 & E13.0 & & & 0.0670 & 0.2191 & 0.1031 & 33.765 \\
\hline Оิ & E13.5 & & & 0.0940 & 0.9229 & 0.1634 & 2.8626 \\
\hline$\sqrt{n}$ & E14.0 & & & 0.0757 & 0.4469 & 0.1068 & 0.4630 \\
\hline
\end{tabular}

Table 5: Comparison is based on four $\mathrm{Hu}$ moments distance methods $d_{1}$ to $d_{4}$. The real and the corresponding simulated images (at each growth time) are the inputs for the match function. Computation for the epithelium EC areas and skeletons is performed in simulation model II (Fig. 9), which is specified in the column description. RI and SI are real images and simulated images, respectively.

across Domains for Word Sense Disambiguation., RANLP, 2017, pp. 1-8.

[2] Affolter, M., Bellusci, S., Itoh, N., Shilo, B., Thiery, J.-P., and Werb, Z., Tube or not tube: remodeling epithelial tissues by branching morphogenesis., Developmental cell, Vol.4, No.1, 2003, pp. 11-8.

[3] Andasari, V., Roper, R. T., Swat, M. H., and Chaplain, M. A., Integrating intracellular dynamics using CompuCell3D and Bionetsolver: applications to multiscale modelling of cancer cell growth and invasion, PloS one, Vol.7, No.3, 2012.

[4] Bao, M., Guo, S., Tang, Q., and Zhang, F., Optimization of the bwmorph Function in the MATLAB image processing toolbox for binary skeleton computation, In 2009 International Conference on Computational Intelligence and Natural Computing IEEE, Vol.2, 2009, pp. 273-276. 


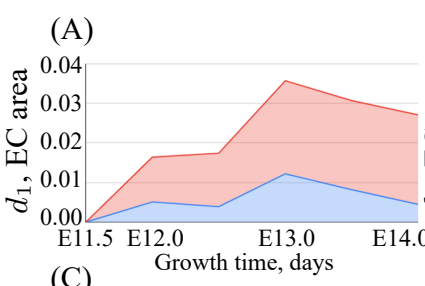

(B)
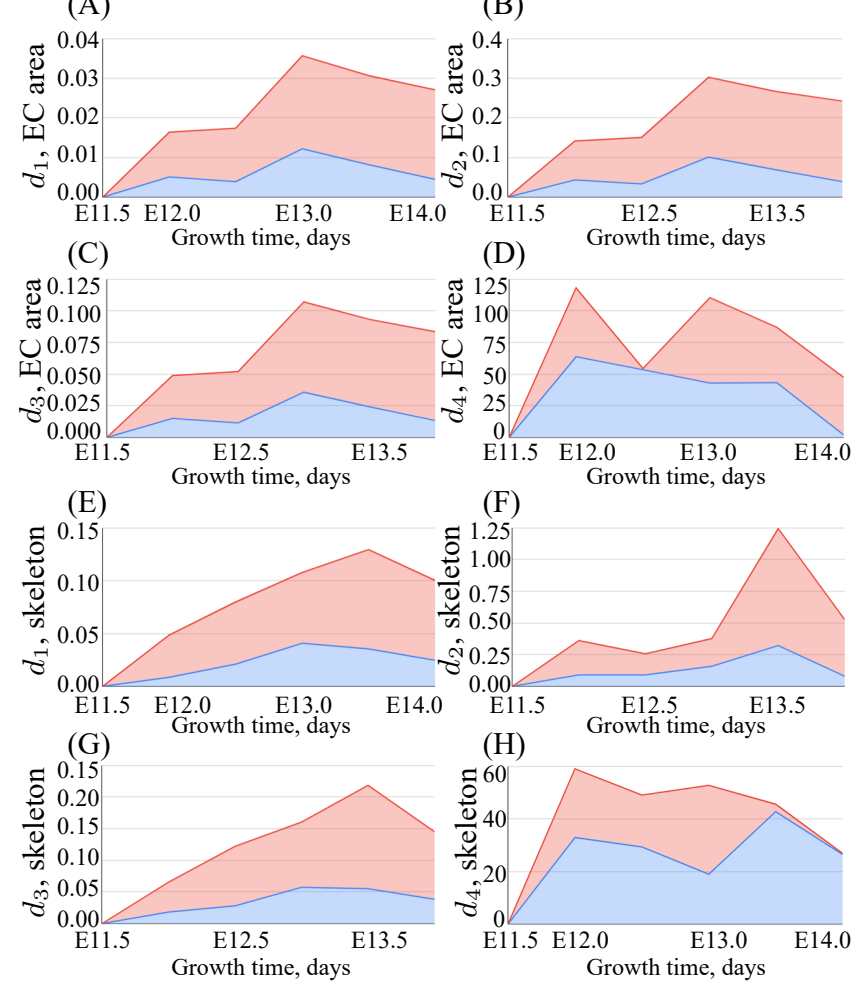

Fig. 10: Effect of different models for kidney branching and the matching distance of simulation model with relevant real biological images. (A-H) simulation results in two models (models I and II) with different distance methods $d_{1}$ to $d_{4}$ ) and related to EC area (A-D) and skeleton (E-H). In each panel, the red lines (and below the surfaces) indicate the distance in model II (Fig. 9) and the blue lines (and below the surfaces) in model I (Fig. 7).

[5] Bentley, K., Mariggi, G., Gerhardt, H., and Bates, P. A., Tipping the balance: robustness of tip cell selection, migration and fusion in angiogenesis, PLoS computational biology, Vol.5, No.10, 2009, pp. e1000549.

[6] Bertram, J. F., Douglas-Denton, R. N., Diouf, B., Hughson, M. D., and Hoy, W. E., Human nephron number: implications for health and disease, $\mathrm{Pe}$ diatric nephrology, Vol.26, No.9, 2011, pp. 1529.

[7] Buzbas, E. O. and Rosenberg, N. A., AABC: approximate approximate Bayesian computation for inference in population-genetic modelsl learning for audio-visual emotion recognition, Theoretical population biology, Vol.99, 2015, pp. 31-42.

[8] Cebrian, C., Asai, N., DAgati, V., and Costantini, F, The number of fetal nephron progenitor cells limits ureteric branching and adult nephron endowment, Cell reports, Vol.7, No.1, 2014, pp. 127-137.
[9] Chen, N., Glazier, J. A., Izaguirre, J. A., and Alber, M. S., A parallel implementation of the Cellular Potts Model for simulation of cell-based morphogenesis, Computer physics communications, Vol.176, No.11-12, 2007, pp. 670-681.

[10] Clément, R. and Mauroy, B., An archetypal mechanism for branching organogenesis, Physical Biology, Vol.11, No.1, 2014, pp. 016003.

[11] Combes, A. N., Towards a quantitative model of kidney morphogenesis, Nephrology, Vol.20, No.5, 2015, pp. 312-314.

[12] Costantini, F., Genetic controls and cellular behaviors in branching morphogenesis of the renal collecting system, Wiley Interdisciplinary Reviews: Developmental Biology, Vol.1, No.5, 2012, pp. 693-713.

[13] Costantini, F. and Shakya, R., GDNF/Ret signaling and the development of the kidney, Bioessays, Vol.28, No.2, 2006, pp. 117-127.

[14] Davis, T. K., Hoshi, M., and Jain, S., To bud or not to bud: the RET perspective in CAKUT, $\mathrm{Pe}$ diatric Nephrology, Vol.29, No.4, 2014, pp. 597608.

[15] Dehling, H., Matsui, M., Mikosch, T., Samorodnitsky, G., and Tafakori, L., Distance covariance for discretized stochastic processes, Bernoulli, Vol.26, No.4, 2020, pp. 2758-2789.

[16] Doerr, F. J. and Florence, A. J., A micro-XRT image analysis and machine learning methodology for the characterisation of multi-particulate capsule formulations, International journal of pharmaceutics: X, Vol.2, 2020, pp. 100041.

[17] Farhoudi, Z. and Setayeshi, S., Fusion of deep learning features with mixture of brain emotional learning for audio-visual emotion recognition, Speech Communication, Vol.127, 2021, pp. 92103.

[18] Gerlee, P. and Anderson, A. R., The evolution of carrying capacity in constrained and expanding tumour cell populations, Physical biology, Vol.12, No.5, 2015, pp. 056001.

[19] Grogan, J. A., Markelc, B., Connor, A. J., Muschel, R. J., Pitt-Francis, J. M., Maini, P. K., and Byrne, H. M., Predicting the influence of microvascular structure on tumor response to radiotherapy, IEEE Transactions on Biomedical Engineering, Vol.64, No.3, 2016, pp. 504-511. 
[20] Hartman, H. A., Lai, H. L., and Patterson, L. T., Cessation of renal morphogenesis in mice, Developmental biology, Vol.310, No.2, 2007, pp. 379387.

[21] Hellmich, H. L., Kos, L., Cho, E. S., Mahon, K. A., and Zimmer, A., Embryonic expression of glial cell-line derived neurotrophic factor (GDNF) suggests multiple developmental roles in neural differentiation and epithelialmesenchymal interactions, Mechanisms of development, Vol.54, No.1, 1996, pp. 95-105.

[22] Hirashima, T., Iwasa, Y., and Morishita, Y., Dynamic modeling of branching morphogenesis of ureteric bud in early kidney development., Journal of theoretical biology, Vol.259, No.1, 2009, pp. 58-66.

[23] James, M. T., Pannu, N., Hemmelgarn, B. R., Austin, P. C., Tan, Z., McArthur, E., Manns, B. J., Tonelli, M., Wald, R., Quinn, R. R., et al., Derivation and external validation of prediction models for advanced chronic kidney disease following acute kidney injury, Jama, Vol.318, No.18, 2017, pp. 1787-1797.

[24] Kicheva, A., Pantazis, P., Bollenbach, T., Kalaidzidis, Y., Bittig, T., Jülicher, F., and Gonzalez-Gaitan, M., Kinetics of morphogen gradient formation, Science, Vol.315, No.5811, 2007, pp. 521-525.

[25] Lambert, B., MacLean, A. L., Fletcher, A. G., Combes, A. N., Little, M. H., and Byrne, H. M., Bayesian inference of agent-based models: a tool for studying kidney branching morphogenesis, Journal of mathematical biology, Vol.76, No.7, 2018, pp. 1673-1697.

[26] Little, M. H. and McMahon, A. P., Mammalian kidney development: principles, progress, and projections., Cold Spring Harbor perspectives in biology, Vol.4, No.5, 2012. doi:10.1101/cshperspect.a008300.

[27] Macklin, P., Edgerton, M. E., Thompson, A. M., and Cristini, V., Patient-calibrated agent-based modelling of ductal carcinoma in situ (DCIS): from microscopic measurements to macroscopic predictions of clinical progression, Journal of theoretical biology, Vol.301, 2012, pp. 122-140.

[28] Menshykau, D. and Iber, D., Kidney branching morphogenesis under the control of a ligandreceptor-based Turing mechanism, Physical biology, Vol.10, No.4, 2013, pp. 046003.
[29] Menshykau, D., Michos, O., Lang, C., Conrad, L., McMahon, A. P., and Iber, D., Image-based modeling of kidney branching morphogenesis reveals GDNF-RET based Turing-type mechanism and pattern-modulating WNT11 feedback, $\mathrm{Na}$ ture communications, Vol.10, No.1, 2019, pp. 239.

[30] Montero, E., Riff, M., and Neveu, B., A beginner's guide to tuning methods, Applied Soft Computing, Vol.17, 2014, pp. 39-51.

[31] Pachnis, V., Mankoo, B., and Costantini, F., Expression of the c-ret proto-oncogene during mouse embryogenesis, Development, Vol.119, No.4, 1993, pp. 1005-1017.

[32] Park, M., Vinaroz, M., and Jitkrittum, W., ABCDP: Approximate Bayesian Computation Meets Differential Privacy, Entropy, Vol.23, No.8, 2021, pp. 961.

[33] Pathmanathan, P., Cooper, J., Fletcher, A., Mirams, G., Murray, P., Osborne, J., Pitt-Francis, J., Walter, A., and Chapman, S., A computational study of discrete mechanical tissue models, Physical biology, Vol.6, No.3, 2009, pp. 036001.

[34] Perfahl, H., Hughes, B. D., Alarcón, T., Maini, P. K., Lloyd, M. C., Reuss, M., and Byrne, H. M., 3D hybrid modelling of vascular network formation, Journal of theoretical biology, Vol.414, 2017, pp. 254-268.

[35] Qiao, J., Sakurai, H., and Nigam, S. K., Branching morphogenesis independent of mesenchymalepithelial contact in the developing kidney., Proceedings of the National Academy of Sciences of the United States of America, Vol.96, No.13, 1999, pp. 7330-5.

[36] Riccio, P., Cebrian, C., Zong, H., Hippenmeyer, S., and Costantini, F., Ret and Etv4 promote directed movements of progenitor cells during renal branching morphogenesis, PLoS biology, Vol.14, No.2, 2016, pp. e1002382.

[37] Schwartz, L., da Veiga Moreira, J., and Jolicoeur, M., Physical forces modulate cell differentiation and proliferation processes, Journal of cellular and molecular medicine, Vol.22, No.2, 2018, pp. 738-745.

[38] Scott, J. G., Hjelmeland, A. B., Chinnaiyan, P., Anderson, A. R., and Basanta, D., Microenvironmental variables must influence intrinsic phenotypic parameters of cancer stem cells to affect tumourigenicity, PLoS computational biology, Vol.10, No.1, 2014, pp. e1003433. 
[39] Short, K. M., Combes, A. N., Lefevre, J., Ju, A. L., Georgas, K. M., Lamberton, T., Cairncross, O., Rumballe, B. A., McMahon, A. P., Hamilton, N. A., et al., Global quantification of tissue dynamics in the developing mouse kidney, Developmental cell, Vol.29, No.2, 2014, pp. 188-202.

[40] Smeeton, J., Dhir, P., Hu, D., Feeney, M. M., Chen, L., and Rosenblum, N. D., Integrin-linked kinase controls renal branching morphogenesis via dual specificity phosphatase 8 , Journal of the American Society of Nephrology, Vol.27, No.5, 2016, pp. 1465-1477.

[41] Szabó, A. and Merks, R. M., Cellular potts modeling of tumor growth, tumor invasion, and tumor evolution, Frontiers in oncology, Vol.3, 2013, pp. 87.

[42] Varner, V. D. and Nelson, C. M., Cellular and physical mechanisms of branching morphogenesis., Development (Cambridge, England), Vol.141, No.14, 2014, pp. 2750-9.

[43] Watanabe, T. and Costantini, F., Real-time analysis of ureteric bud branching morphogenesis in vitro, Developmental biology, Vol.271, No.1, 2004, pp. 98-108.

[44] Yu, S. R., Burkhardt, M., Nowak, M., Ries, J., Petrášek, Z., Scholpp, S., Schwille, P., and Brand, M., Fgf8 morphogen gradient forms by a sourcesink mechanism with freely diffusing molecules, Nature, Vol.461, No.7263, 2009, pp. 533.

[45] Zhang, Y., Wang, S., Sun, P., and Phillips, P., Pathological brain detection based on wavelet entropy and $\mathrm{Hu}$ moment invariants, Bio-medical materials and engineering, Vol.26, No.s1, 2015, pp. S1283-S1290.

[46] Zubkov, V., Combes, A. N., Short, K., Lefevre, J., Hamilton, N. A., Smyth, I., Little, M. H., and Byrne, H. M., A spatially-averaged mathematical model of kidney branching morphogenesis, Journal of theoretical biology, Vol.379, 2015, pp. 2437.

[47] Žunić, J., Hirota, K., and Rosin, P. L., A Hu moment invariant as a shape circularity measure, Pattern Recognition, Vol.43, No.1, 2010, pp. 4757.

\section{Contribution of individual authors to the creation of a scientific article (ghostwriting policy)}

Author Contributions: Please, indicate the role and the contribution of each author:

Example

Prof. Dr. Karl-Heinz Zimmermann has supervised and led the project.

Afshin Poorkhanalikoudehi has done the research and implemented the algorithms.

Follow: www.wseas.org/multimedia/contributorrole-instruction.pdf

\section{Sources of funding for research presented in a scientific article or scientific article itself}

Report potential sources of funding if there is any

\section{Creative Commons Attribution License 4.0 (Attribution 4.0 International, CC BY 4.0)}

This article is published under the terms of the Creative Commons Attribution License 4.0

https://creativecommons.org/licenses/by/4.0/deed.en US 